\title{
Delay-throughput analysis of multi-channel MAC protocols in ad hoc networks
}

\author{
Jari Nieminen ${ }^{*}$ and Riku Jäntti
}

\begin{abstract}
Since delay and throughput are important Quality of Service parameters in many wireless applications, we study the performance of different multi-channel Media Access Control (MAC) protocols in ad hoc networks by considering these measures in this paper. For this, we derive average access delays and throughputs in closedform for different multi-channel MAC approaches in case of Poisson arrivals. Correctness of theoretical results is verified by simulations. Performance of the protocols is analyzed with respect to various critical operation parameters such as number of available channels, packet size and arrival rate. Presented results can be used to evaluate the performance of multi-channel MAC approaches in various scenarios and to study the impact of multichannel communications on different wireless applications. More importantly, the derived theoretical results can be exploited in network design to ensure system stability.
\end{abstract}

\section{Introduction}

Multi-channel communications form the basis of various future wireless systems such as cognitive radio, next generation cellular and wireless sensor networks (WSNs). The reason for this is that the performance of a wireless network can be improved by exploiting multiple frequency channels simultaneously to ensure robustness, minimize delay and/or enhance throughput. In general, performance of multi-channel networks is heavily dependent upon used Media Access Control (MAC) protocols and efficient medium access schemes are considered as an essential part of any power-limited selfconfigurable wireless ad hoc network [1]. Furthermore, delay and throughput are important Quality of Service (QoS) parameters in many applications [2] and hence, the performance of multi-channel MAC schemes in ad hoc networks should be investigated in detail with respect to these measures.

In the case of single-channel systems, the performances of various MAC approaches have been investigated by considering both, throughput and delay. Carrier Sense Multiple Access (CSMA) for single channel systems was first studied by Kleinrock and Tobagi in [3], where the authors deduced equations for delays and throughputs of CSMA and ALOHA using the busy

\footnotetext{
* Correspondence: jari.nieminen@aalto.fi

Department of Communications and Networking, School of Electrical Engineering, Aalto University, P.O. Box 13000, 00076 Aalto, Finland
}

period analysis. Later on delay distributions of slotted ALOHA and CSMA systems were derived in [4] for different retransmission methods. Operation of singlechannel IEEE 802.11 systems was evaluated in [5] comprehensively using a Markov chain model to model the impact of backoff window sizes on the performance. Multi-channel MAC approaches have not been studied as widely but a performance analysis of different multichannel protocols in a single collision domain was presented in [6] with respect to data rates by assuming saturated traffic conditions. However, to the best of authors' knowledge, delay-throughput characteristics of multi-channel MAC protocols have not been studied yet in case of Poisson arrivals and infinite number of users.

Contention-based multi-channel MAC protocols designed for ad hoc networks can be divided into three main classes, namely split phase, periodic hopping and dedicated control channel. In split phase-based random access approaches the operation is divided into two parts. First, during contention periods nodes reserve resources on a common control channel and afterwards, data transmissions will take place during the data period. On the other hand, the basic idea behind periodic hopping approaches is to use channel hopping on every channel to avoid availability and congestion problems of the common control channel. Moreover, dedicated control channel schemes allocate one channel as a common control channel and carry out data transmissions on 
other channels. Each of these approaches has specific strengths and weaknesses which will be discussed in detail.

In this paper, we derive average access delays and throughputs for different multi-channel MAC approaches in case of Poisson arrivals and analyze the performance with respect to delay and throughput. We use a similar approach as in [4] but extend the analysis by taking into account the effect of multi-channel communications and deduce the closed-form solutions for different multi-channel MAC schemes. Correctness of theoretical derivations will be attested by simulations. Performance of the protocols is then analyzed with respect to various critical operation parameters such as number of available channels, packet size and arrival rate. Presented results can be used to analyze the performance and suitability of different multi-channel MAC approaches for prospective wireless applications and to guide system design.

The rest of the paper is organized as follows. In Section II, we specify used system models. Next, we introduce different multi-channel approaches in Section III and derive throughputs and expected delays in Section IV for the different multi-channel protocols. Results and analyses are presented in Section V. Section VI summarizes the paper.

\section{System model}

In this paper, the focus is on MAC in multi-channel ad hoc networks. Since optimal FDMA/TDMA schemes introduce a lot of complexity and additional messaging, we restrict our study to random access schemes. Each device is equipped with one half-duplex transceiver which makes protocols that require an additional receiver, such as [7], impracticable. Throughputs and delays of different contention-based multi-channel MACs can be modeled similarly to single-channel CSMA systems with the exception that now we have multiple channels to be exploited. We presume that a common control channel (CCC) is predetermined for the protocols that require a CCC for functioning and it is always of good quality.

If a packet transmission fails for some reason, retransmission of the packet will be attempted until successful transmission takes place, i.e. packets will not be discarded in any case. For the analysis, we divide the operation into multiple discrete time slots and assume fixed packet sizes along with perfect time synchronization among the nodes. The length of a time slot $\tau$ is defined to correspond to the maximum propagation delay of resource request and acknowledgement messages. Channel sensing time is equal to the maximum propagation delay as well and we neglect channel switching penalty for the sake of simplicity. We only consider slotted systems with an infinite number of users.

Packet arrivals are modeled as a Poisson process with rate $g$ packets per time slot which includes both, new and retransmitted, packet arrivals. In the case of retransmissions, we consider large backoff windows, e.g. $\omega>20$ such as in [4]. Thus, a station generates one packet in a given time slot $(t, t+\tau)$ with probability

$$
P[N(t+\tau)-N(t)=1]=\mathrm{e}^{-g \tau}(g \tau),
$$

where $N(t)$ is the number of occured events up to time $t$. All new packets will try to access the channel in the following time slot immediately after generation. Furthermore, we assume fixed packet sizes with transmission time $T$ and define $2 \tau<T$. Packet transmission time $T$ also includes the acknowlegment message from the receiver. All the nodes in a network are awake constantly and have identical channel conditions.

\section{Multi-Channel MAC protocols in ad hoc networks}

Research efforts in the field of access mechanisms for single-channel ad hoc networks have been extensive. For example, a multiplicity of single-channel MAC protocols has been proposed for WSNs [8]. Moreover, various multi-channel MAC protocols have been designed for different wireless systems as well. In this section, we briefly introduce operation principles of the most popular multi-channel MAC approaches for which average access delays and throughputs will be derived in Section IV. We divide random access multi-channel MACs into three main categories based on the nature of operations: split phase, periodic hopping and dedicated control channel. The categories include several protocols designed for different purposes of use such as Cognitive Radio Network (CRN), WSN and Wireless Local Area Network (WLAN). We will choose only one protocol from each category for a detailed study. In all of the considered cases resource reservations and negotiations are based on the IEEE 802.11 RTS/CTS message exchange. The main problem of multi-channel systems is the multi-channel hidden node problem which occurs if the channel usage of neighbor nodes is not known and nodes choose to transmit on a busy channel.

In split phase approaches the operation is divided into two parts. First, during contention periods nodes reserve resources on the chosen common control channel and then data transmissions will take place during data periods. Split phase approach has been proposed in different contexts. For example, in WLANs Multi-channel MAC (MMAC) protocol [9] exploits this approach whereas in case of CRNs Cognitive MAC (C-MAC) [10] uses similar frame structure. From these we choose MMAC, 
since it is designed for general ad hoc networks, for our delay-throughput analysis. Operation of MMAC is illustrated in Figure 1a.

Periodic hopping protocols hop on all the channels according to a hopping pattern to avoid availability and congestion problems of the common control channel. Nodes may obey a common hopping pattern or have individual hopping patterns. In multi-channel WLANs the common hopping approach is used for example in Channel-Hopping Multiple Access (CHMA) which was introduced in [11]. In addition, in the context of CRNs at least SYN-MAC [12] uses this approach and similar approach has been proposed for WSNs as well, called Y-MAC [13], which starts hopping only in the case of congestion. McMAC [14] and Slotted Seeded Channel Hopping (SSCH) [15] are examples of protocols which employ individual hopping patterns. Since the delaythroughput performance of various periodic hopping protocols is similar, we select SYN-MAC and evaluate its performance in this paper. Functioning of SYN-MAC is depicted in Figure 1b.
Dedicated control channel approaches use one channel only for distributing control information. The idea was first presented in [16], where the basic operation of IEEE 802.11 was extended for multiple channels simply by allocating data transmissions to different channels. However, the multi-channel hidden node problem is completely ignored in the design. A protocol which considers the multi-channel hidden node problem in this class is CAM-MAC [17]. CAM-MAC requires all neighbors that hear a resource request message to verify availability of the proposed data channel. Consequently, channel reservations consume a lot of resources.

A dedicated control channel approach which consumes less resources than CAM-MAC while considers the multi-channel hidden node problem is Generic Multi-channel MAC (G-McMAC) [18]. Thus, we choose G-McMAC for the analysis from this class. The protocol is designed especially for multi-channel WSNs. GMcMAC is a hybrid CSMA/TDMA protocol in which contention and data periods are merged to minimize delays. In general, the operation of the protocol is

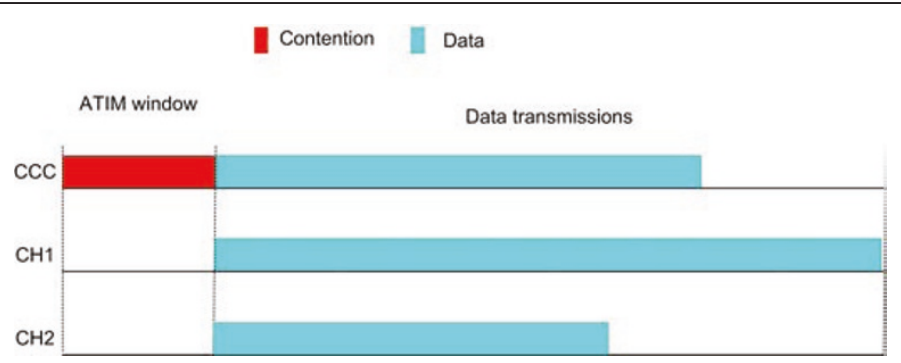

(a) Split phase: Multi-channel MAC (MMAC)

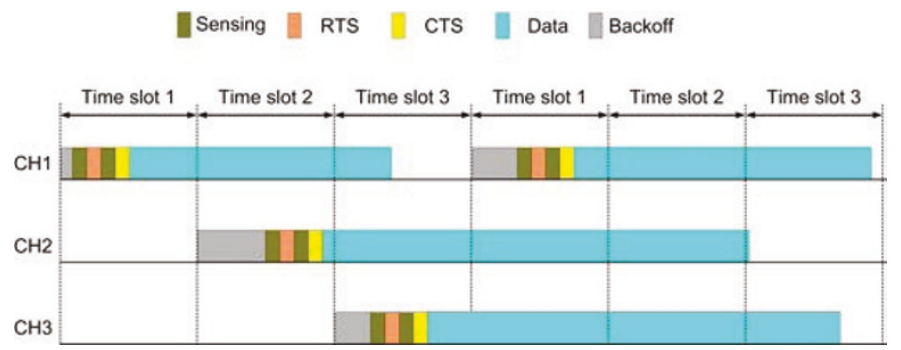

(b) Periodic hopping: Synchronized MAC (SYN-MAC)

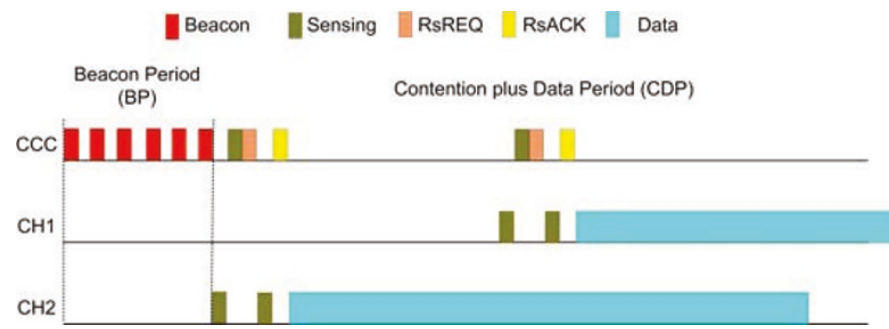

(c) Dedicated control channel: Generic Multi-channel MAC (G-McMAC)

Figure 1 Multi-channel MAC approaches. (a) Split phase: Multi-channel MAC (MMAC). (b) Periodic hopping: Synchronized MAC (SYN-MAC). (c) Dedicated control channel: Generic Multi-channel MAC (G-McMAC). 
divided into two segments: Beacon Period (BP) and Contention plus Data Period (CDP). Activities of GMcMAC are illustrated in Figure 1c.

Each beacon includes the following information: preferable channel list, send time stamp, channel schedules, hierarchy level, beacon interval length. Gateway node $(\mathrm{GW})$ of the WSN is on level 1 on the synchronization hierarchy and starts the beaconing process by sending the first beacon. All the receivers synchronize to the time reference provided by the GW and set their level as 2 . After this, the nodes on level 2 will broadcast beacons as well and so forth. After a node has received beacons from all its neighbors, it can start the data negotiation process. If a node has a packet to send it first senses the wanted data channel to acquire the latest channel information and after this the node will send a Resource Request (RsREQ) message to the intended receiver which includes the desired data channel and transmission time, if the channel is free. The proposed frequency-time block will be chosen by utilizing the receiver's and transmitter's preferable channel lists and schedules. After receiving a RsREQ message, the intended receiver will sense the desired data channel and respond with a Resource Acknowledgment (RsACK) message on the common control channel if the proposed channel is available. Afterwards, the nodes will carry out the data transmission on the chosen channel at the agreed time.

\section{Throughputs and expected delays}

Since, we assume that packet arrivals follow Poisson process performance evaluation of random access schemes can be carried out by exploiting the busy period analysis [19], where the average busy time $\bar{B}$ and average idle time $\bar{I}$ are used for determining the characteristics of various schemes. In the appendices, we derive the following probabilities for different multichannel protocols using the busy period analysis: $P_{\mathrm{s}}$ is the probability of successful transmission, $P_{\mathrm{c}}$ is the probability of collision and $P_{\mathrm{b}}$ is the probability that the channel is sensed busy. In this section, we derive closedform solutions for average access delays and throughputs of various multi-channel MAC schemes individually by exploiting derived probabilities. Theoretical results are confirmed by simulations. Examined protocols are G-McMAC, MMAC and SYN-MAC. In the case of G-McMAC and SYN-MAC, we derive the theoretical results rigorously. On the other hand, since MMAC uses finite contention windows, only approximations can be found in case of MMAC which are then justified by simulation results.

We specify throughput $S$ as follows

$$
S=g T P_{S}
$$

where $T$ is the packet size in discrete time slots $\tau$. Next, we define average access delay. Average access delay is the sum of the initial access delay and the delay because of $i$ unsuccessful transmissions. We denote the initial access delay with $D_{0}$ and the delay of $i$ th retransmission with $D_{i}$. In general form, the total access delay in case of $R$ retransmissions is

$$
D=\sum_{i=0}^{R} D_{i}
$$

Added to this, different retransmission policies induce different delays. By denoting the $i$ th backoff delay as uniformly distributed random variable $W_{i} \sim U\left(1,2^{i-1} \omega\right)$, where $\omega$ is the original backoff window in slots, we get the following expected delay for the $i$ th backoff in case of Binary Exponential Backoff (BEB)

$$
E\left[W_{i}^{\mathrm{beb}}\right]=\frac{1+2^{i-1} \omega}{2}
$$

and, respectively, in case of Uniform Backoff (UB) we have $W_{i} \sim U(1, \omega)$ and

$$
E\left[W^{\mathrm{ub}}\right]=\frac{1+\omega}{2} \text {. }
$$

\section{A. Generic multi-channel MAC (G-McMAC)}

First, we derive equations for the throughput and average access delay of G-McMAC [18]. We exclude the beacon period from this analysis since beaconing may be used by other protocols as well, such as MMAC, or periodic beaconing may be required for time synchronization, routing, etc. For example, many routing protocols use broadcast messages to distribute routing information [20] and hence, require a beacon period in practice to avoid transmission of a routing packet many times. Moreover, it is presumed that beacon periods are carried out rarely such that the impact of the period is negligible to the packet arrival process.

In G-McMAC, if a node generates a packet, it first senses the desired data channel to make sure that it is idle. After this, if the channel is sensed busy, a random backoff will be induced. On the other hand, if the desired channel is free the RTS/CTS message exchange will be carried out on the CCC. The receiver will sense the wanted data channel before replying. Finally, if the message exchange was performed successfully, nodes can start the data transmission on the chosen data channel. Figure 2 illustrates the operation of G-McMAC during CDPs in detail.

Average access delay of G-McMAC depends on two issues. First, the contention process on the CCC and possible collisions induce some delay. Second, if all data 


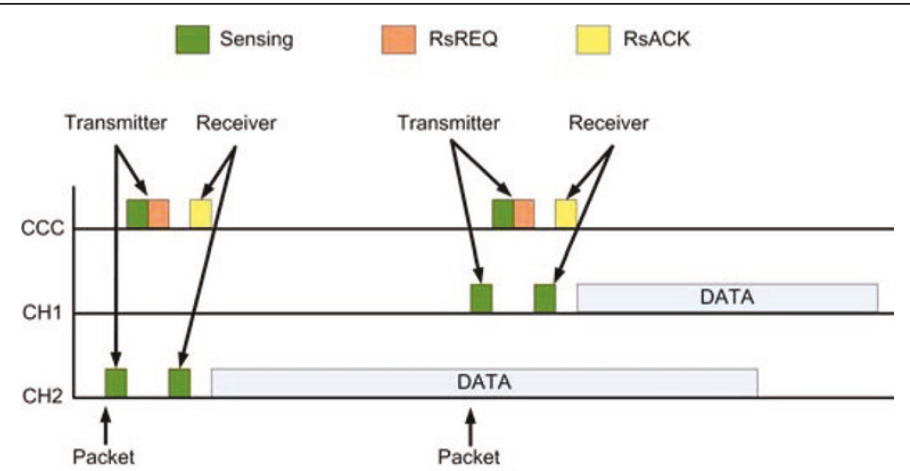

Figure 2 Operation of G-MCMAC during a CDP.

channels are occupied an extra delay will be added as well. We deduce the delay of the contention process first and the impact of occupied channels will be taken into account while deriving the probability of successful transmission in Appendix A.

Now, if the CCC is sensed busy, the latency time for the first unsuccessful transmission is

$$
D_{1}=\left(W_{1}+1\right) \cdot \tau \text {. }
$$

Respectively, if the channel is sensed idle, the transmitter waits for $4 \tau$ to conclude whether there was a collision or not. In case of a collision, the delay time for the second unsuccessful transmission is

$$
D_{2}=\left(W_{2}+4\right) \cdot \tau \text {. }
$$

Consequently, by denoting the number of retransmission because of the channel is sensed busy by $K$, the total delay time can be calculated as follows

$$
\begin{aligned}
D & =D_{0}+\sum_{i=1}^{K}\left(W_{1}+1\right) \cdot \tau+\sum_{j=K+1}^{R}\left(W_{2}+4\right) \cdot \tau \\
& =D_{0}+\tau \sum_{i=1}^{R} W_{i}+K \tau+4(R-K) \cdot \tau,
\end{aligned}
$$

where $D_{0} \sim U(5 \tau, 6 \tau)$ is the initial transmission delay in case of successful transmission and $0 \leq K \leq R$. Hence, $R-K$ is the amount of retransmission due to packet collisions during contention. The joint distribution of $R$ and $K$ is

$$
P\{R=r, K=k\}=\left(\begin{array}{l}
r \\
k
\end{array}\right) P_{\mathrm{b}}^{k} P_{\mathrm{c}}^{r-k} P_{\mathrm{s}}, \quad 0 \leq k \leq r,
$$

and the used probabilities for G-McMAC are derived in Appendix A. Now, the expected delay conditioned on $R=r$ and $K=k$ for G-McMAC can be formulated as

$$
\begin{aligned}
E[D \mid R=r, K=k]= & E\left[D_{0}\right]+\tau \sum_{i=1}^{r} E\left[W_{i}\right] \\
& +k \tau+4(r-k) \cdot \tau \\
& =\frac{\tau}{2}\left(\omega 2^{r}+9 r-6 k+11-\omega\right) .
\end{aligned}
$$

Moreover, to derive the average access delay we need to remove the conditioning on $R$ and $K$. Therefore, the average access delay is given by

$$
\begin{aligned}
\bar{D}= & \sum_{r=0}^{\infty} \sum_{k=0}^{r} E[D-R=r, K=k] \cdot P\{R=r, K=k\} \\
= & \frac{\tau}{2}\left(\frac{\omega P_{\mathrm{s}}}{1-2\left(1-P_{\mathrm{s}}\right)}+\frac{9}{P_{\mathrm{s}}}-\frac{6 P_{\mathrm{b}}}{P_{\mathrm{s}}}+2-\omega\right), \\
& P_{\mathrm{s}}>0.5,
\end{aligned}
$$

where $P_{\mathrm{s}}>0.5$ is required to have a finite average delay. In addition, availability of channels causes additional delay as well. We model the impact of multichannel communications using a Markov model and thus, the probability that all the data channels are occupied $\left(P_{\text {occ }}\right)$ can be calculated using the Erlang B formula [21]. As a result, the throughput of G-McMAC is

$$
S=g T \cdot \frac{e^{-g \tau}}{4-3 e^{-g \tau}} \cdot\left(1-P_{\text {occ }}\right)
$$

where

$$
P_{\mathrm{occ}}=\frac{\frac{G^{N-1}}{(N-1) !}}{\sum_{i=0}^{N-1} \frac{G^{i}}{i !}}
$$

and $G=g T$. Since the control channel is not used for data transmissions, only $N-1$ channels are available for data transmissions. Figure 3 shows that the theoretical 


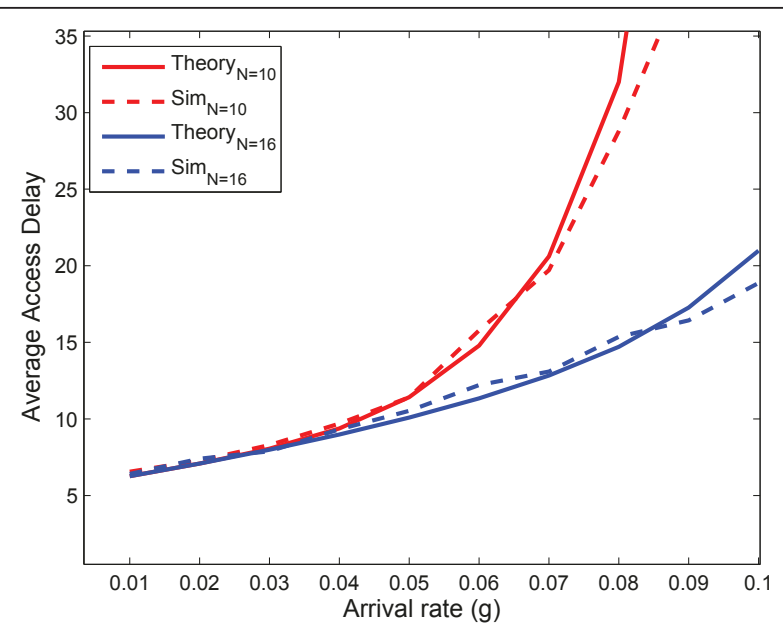

Figure 3 Theoretical and simulated results for average access delay of G-McMAC $(T=100, \omega=32)$.

and simulated results match up well for different number of channels with respect to delay.

\section{B. Multi-channel MAC (MMAC)}

Next, we study split phase approaches using MMAC [9] as an example. Operation of MMAC is divided into two parts which form a cycle. MMAC is designed for IEEE 802.11 networks and it exploits Ad hoc Traffic Indication Message (ATIM) windows of IEEE 802.11 Power Saving Mechanism (PSM) which are originally used only for power management. In MMAC ATIM windows are extended and channel reservations conducted during ATIM windows on the CCC. Data transmissions take place on all available channels afterwards. We denote the length of the ATIM window by $T_{\text {atim }}$ and the length of the data interval by $T$, both in time slots. Thus, the total length of one cycle is $T_{\mathrm{c}}=T_{\mathrm{atim}}+T$. Lengths of these intervals are predetermined and fixed and hence, the intervals determine the average access delay as well. We set $T_{\text {atim }}=0.2 \cdot T_{\mathrm{c}}$ and $T=0.8 \cdot T_{\mathrm{c}}$ since these values were used in the initial simulation model in [9].
Furthermore, it is assumed that packets fit perfectly to the chosen cycle structure. Figure 4 depicts the operation of MMAC during ATIM windows.

In the case of MMAC, a node has to wait until the end of an ATIM window even though the initial transmission would be successful before transmitting data. Consequently, on average the initial transmission delay is

$$
E\left[D_{0}\right]=\frac{T_{\text {atim }}}{2} \cdot \frac{T_{\text {atim }}}{T_{\mathrm{c}}}+\left(\frac{T_{D}}{2}+T_{\text {atim }}\right) \cdot \frac{T_{D}}{T_{\mathrm{c}}} .
$$

Moreover, if a node has not been able to reserve resources before the end of an ATIM window, it has to wait for the next data interval and an additional delay of $T_{\mathrm{c}}$ is added. Hence, the overall delay is

$$
D=D_{0}+M \cdot T_{\mathrm{c}}
$$

where $M$ denotes the number of additional cycles. If the delay due to CSMA operations during an ATIM window is larger than the length of the ATIM window or all of the channels are occupied before a node can reserve resources, a packet will be delayed. By denoting the latency of a packet during an ATIM window with $L$, this blocking probability can be represented as

$$
P_{\text {block }}=P\left\{L>T_{\text {atim }}\right\}+P\left\{L \leq T_{\text {atim }}\right\} \cdot P\{\text { Occupied }\} .
$$

Since all resource reservations will be made during ATIM windows, the packet arrival rate has to be scaled such that all packets are generated during an ATIM window in one cycle for theoretical analysis. Hence, in theory we have the following packet arrival rate for the contention phase

$$
g_{a}=g \cdot \frac{T_{\mathrm{c}}}{T_{\mathrm{atim}}} .
$$

First, we find out the probability that a node can not reserve resources during an ATIM window due to the shortage of data channels. We approximate this by comparing the number of channel reservations to the number of channels. This is done by scaling the difference

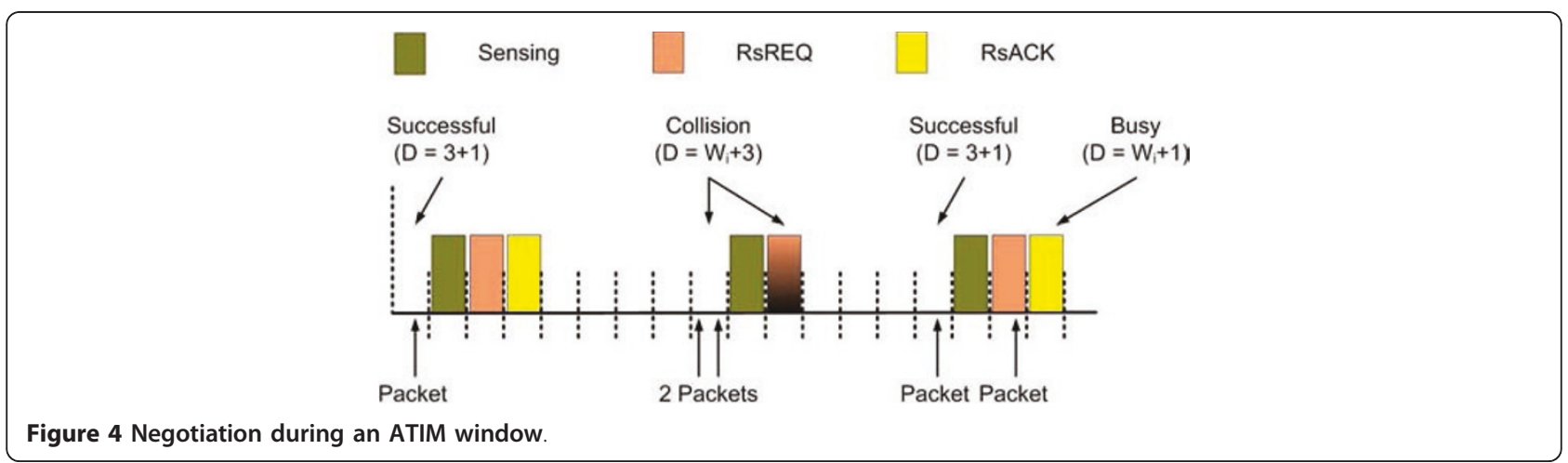


between the amount of successful negotiations and the number of channels with the amount of successful negotiations. All of the used probabilities are derived in Appendix B. The amount of successful data negotiations during an ATIM window is on average

$$
E[\text { packets }]=P_{\mathrm{s}} g_{a} T_{\text {atim }} .
$$

In the beginning of each ATIM window all the channels are free and hence, the previously used Markov model can not be exploited. In consequence, we approximate the probability that a packet is blocked because of channel shortage as follows

$$
P_{\text {b1ock }}^{c} \approx \max \left\{0, \frac{P_{\mathrm{s} g} T_{\mathrm{atim}}-N}{P_{\mathrm{s} g} T_{\mathrm{atim}}}\right\} .
$$

Second, in the case of small ATIM windows, the performance will be bounded by the fact that only a certain amount of data channels can be reserved in time before the end of an ATIM window. Now, if a node senses that the control channel is busy during contention, it will backoff according to BEB. Same happens in case of collisions as well. During an ATIM window the latency of a successful RTS/CTS message exchange is $3 \tau$. Since $\omega=$ 32, the performance is dominated by $P\{R=0\}$ and $P\{R$ $=1\}$ while the total delay is $L \leq 35$. Furthermore, while $35<T_{\text {atim }} \leq 2 \omega, P\{R \leq 2\}$ dominates. Finally, if $T_{\text {atim }}>$ $2 \omega$ the effect of $P_{\text {b1ock }}^{d}$ becomes negligible since multiple retransmissions may take place and it is very unlikely that a packet is delayed due to the end of an ATIM window. We set the probability of a retransmission as $P_{r}=$ $P_{\mathrm{c}}+P_{\mathrm{b}}$ and approximate the probability of block due to the end of a contention window as follows

$$
P_{\text {blockc }}^{d} \approx\left\{\begin{array}{l}
1-\left(P_{\mathrm{s}}+P_{r} \cdot P_{\mathrm{s}} \cdot T_{\mathrm{atim}}\right), \quad T_{\text {atim }} \leq 35, \\
1-\left(P_{\mathrm{s}}+\left(P_{r}^{2}+P_{r}\right) \cdot P_{\mathrm{s}}\right), \quad 35<T_{\text {atim }} \leq 2 \omega, \\
0, \quad \text { otherwise. }
\end{array}\right.
$$

Figure 5 depicts theoretical and simulated results for different packet sizes. When the packet size is 100 , the blocking probability is determined by $P_{\text {b1ock }}^{d}$ and with the packet size of 1,000 , the blocking probability is

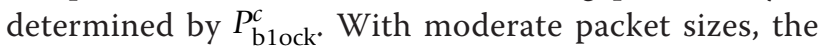
blocking probability is determined by both probabilities and hence, the simulated and theoretical results do not match perfectly. Nevertheless, according to our results these approximations do not significantly under- or overestimate the performance of MMAC in any case and hence, the use of these approximates is justifiable for adequate analysis.

Finally, effect of additional cycles can be formulated as

$$
\bar{D}_{\text {block }}=\left(P_{\text {b1ock }}^{d}+P_{\text {b1ock }}^{c}-P_{\text {b1ock }}^{d} \cdot P_{\text {b1ock }}^{c}\right) T_{\mathrm{c}}
$$

and thus, the average access delay of MMAC is given by

$$
\bar{D}=E\left[D_{0}\right]+\bar{D}_{\text {block}} \text {. }
$$

and the throughput is

$$
S=g_{a} T \cdot \frac{\mathrm{e}^{-g \tau}}{\left(3-2 \mathrm{e}^{-g_{a} \tau}\right)} \cdot\left(1-P_{\text {block }}\right) \text {. }
$$

\section{Synchronized MAC (SYN-MAC)}

We use SYN-MAC [12] as an example of common hopping approaches and the same delay-throughput analysis applies to parallel rendezvous schemes as well. SYNMAC exploits periodic hopping and resource reservations can be done only for the current channel to avoid the multi-channel hidden node problem. Therefore, the performance of SYN-MAC can be estimated similarly to single-channel systems by reducing the arrival rate of packets due to the utilization of multiple channels simultaneously. General operation of SYN-MAC on a single channel is demonstrated in Figure 6. For analysis purposes, we assume that all generated packets have to wait until the next resource reservation interval before competing for resources and data transmissions start precisely at the end of contention windows.

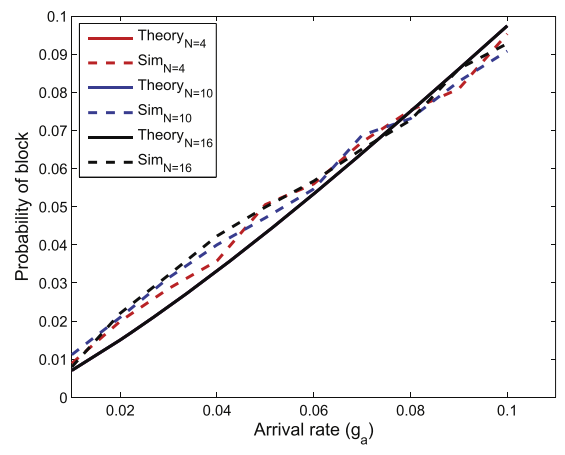

(a) $\mathrm{T}=100\left(P_{\text {block }}^{d}\right.$ dominates)

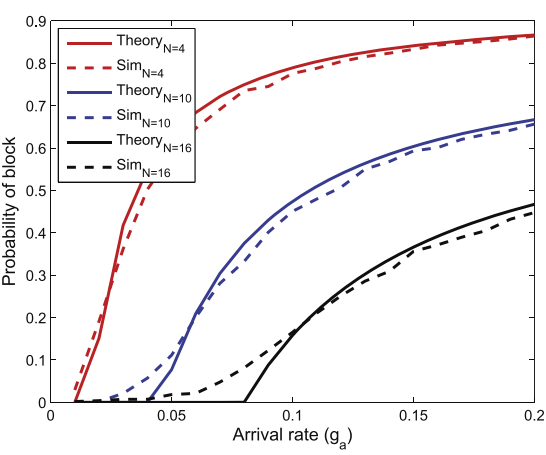

(b) $\mathrm{T}=1000\left(P_{\text {block }}^{c}\right.$ dominates $)$

Figure $\mathbf{5}$ Theoretical and simulated results for the probability of block as a function of arrival rate. (a) $T=100\left(P_{\text {block }}^{d}\right.$ dominates). (b) $T=$ 1,000 ( $P_{\text {block }}^{c}$ dominates). 


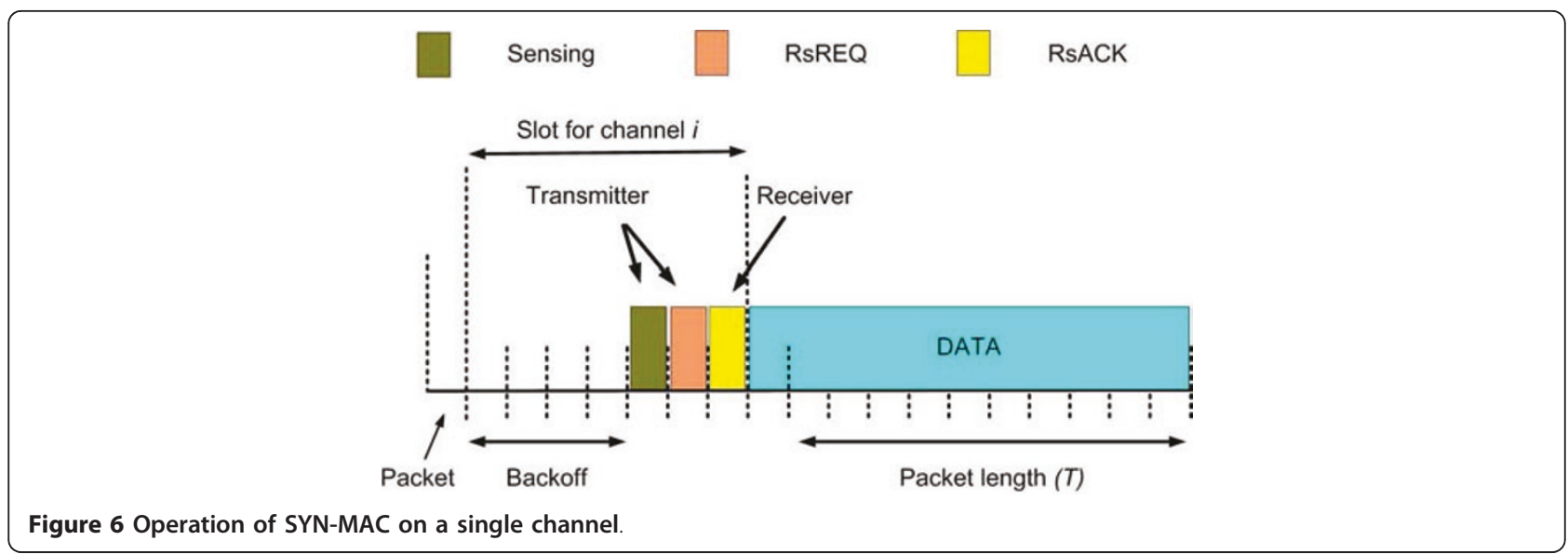

Resource reservation interval is divided into multiple small time slots $(\tau)$ and to avoid collisions, each transmitter chooses a random backoff value from a given fixed window $\omega$. In other words, SYN-MAC exploits UB. Length of the contention period is $T_{\mathrm{s}}=\omega \tau$ and we set $T_{\mathrm{s}}=10$ since this should give good results in general according to [12]. Consequently, to validate the assumption of Poisson arrivals, retransmitted packets are delayed over several contention windows randomly in simulations. Moreover, we denote the total length of a cycle by $T_{\mathrm{c}}=T+T_{\mathrm{s}}$.

Arrival rates have to be scaled correspond to the operation of SYN-MAC. Naturally, the arrival rate is inversely proportional to the number of channels $N$. Moreover, packets generated during the packet transmission time $T$ will stack up. Hence, in case of SYNMAC we scale arrival rates as follows

$$
g_{\mathrm{s}}=g \cdot \frac{\omega+T}{T / \omega} \cdot \frac{1}{N}=g \cdot \frac{(\omega+T) \omega}{T \cdot N} .
$$

Now, in case of SYN-MAC the latency of a successful transmission is simply

$$
E\left[D_{0}\right]=T_{\mathrm{s}}+\frac{T_{\mathrm{s}}}{2}
$$

on average. Contrary to other approaches, the induced latencies because of collisions or if a channel is sensed busy are equal in SYN-MAC. If resource request messages collide or the channel is sensed busy, a delay of $T_{\mathrm{s}}$ will be added always. Thus, the delay due to $R$ retransmissions is simply

$$
D_{r}=\sum_{i=1}^{R} T_{\mathrm{s}}=T_{\mathrm{s}} \cdot R
$$

and the amount of retransmissions on average is given by

$$
E[R]=\frac{P_{\mathrm{b}}+P_{\mathrm{c}}}{P_{\mathrm{s}}} .
$$

Finally, we can find out the average access delay as follows

$$
\begin{aligned}
\bar{D} & =E\left[D_{0}\right]+T_{\mathrm{s}} \cdot E[R] \\
& =E\left[D_{0}\right]+T_{\mathrm{s}}\left(\frac{1-P_{\mathrm{s}}}{P_{\mathrm{s}}}\right) \\
& =T_{\mathrm{s}}\left(\frac{2+P_{\mathrm{s}}}{P_{\mathrm{s}}}\right), P_{\mathrm{s}}>0,
\end{aligned}
$$

and the throughput is

$$
S=g_{\mathrm{s}} T \cdot \frac{\left(T_{\mathrm{s}} / T\right) \mathrm{e}^{-g_{\mathrm{s}} \tau}}{1+\left(T_{\mathrm{s}} / T\right)-\mathrm{e}^{-g_{\mathrm{s}} \tau}} .
$$

The probabilities for SYN-MAC are derived in Appendix C. Again, we compare our theoretical results with simulation results and the outcome is illustrated in Figure 7. With large packets $\left(T \geq(N-1) T_{\mathrm{s}}\right)$ theoretical and simulated results are identical when $P_{\mathrm{s}} \geq 0.5$. But then, with smaller packets $\left(T<(N-1) T_{\mathrm{s}}\right)$ results are slightly different since a data transmission on one channel will be over before nodes hop onto that particular channel again and thus, packet size does not have any impact on the performance in that case. Nevertheless, since the probabilities of successful transmission and that the channel is sensed busy match without using Equation (23) and retransmissions, we conclude that the theoretical results for SYN-MAC are correct.

\section{Results and analysis}

In this section, we analyze the performance of different multi-channel MAC approaches with respect to throughput and average access delay using previously deduced analytical results which were confirmed by simulations. First, we focus on delay analysis and 


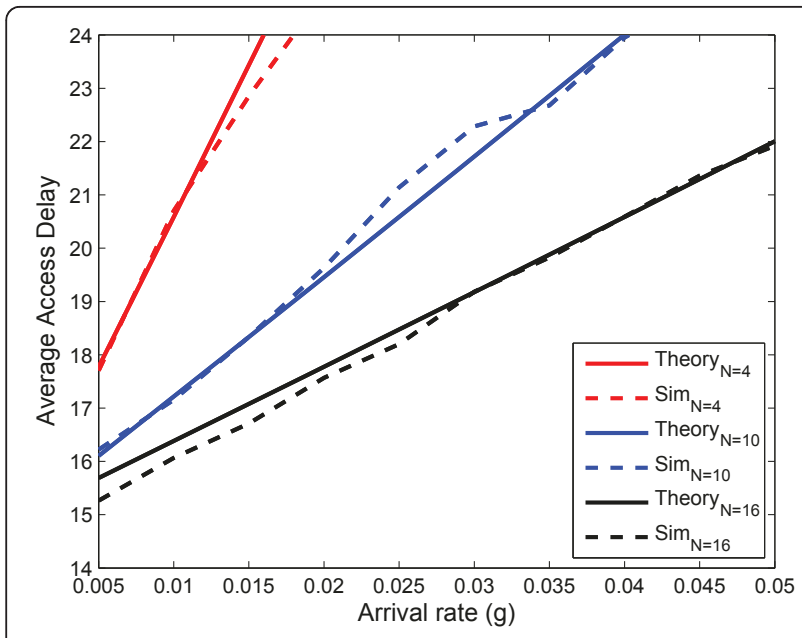

Figure 7 Theoretical and simulated results for average access delay in case of SYN-MAC $(T=200, \omega=10)$.

consider the impact of arrival rate, number of channels and packet sizes on the expected delays. Second, we evaluate the performance of the protocols in terms of total throughput with respect to the same critical system parameters. Finally, we consider stability of the different approaches since it is of significant importance to understand what is the maximum traffic load that a MAC protocol can handle.

\section{A. Delay analysis}

It is extremely important to understand delay characteristics of the used MAC protocols to assure sufficient QoS and system stability. Hence, in this subsection we analyze the performance of different multi-channel MAC protocols with respect to average access delay. First, we consider the effect of packet size and present the results for average access delay as a function of packet size in Figure 8. In general, G-McMAC offers significantly lower delays than other approaches with small packets regardless of the number of channels and the impact of packet size starts to be visible just before approaching the stability point, which is $T=300$ while $N=10$ and $g=0.04$ for G-McMAC, even though the impact of packet size on the delay is small in general. Stability point of G-McMAC, and other protocols as well, moves to the left on $x$-axis if the arrival rate is increased and right if the arrival rate is decreased.

Moreover, SYN-MAC offers relatively constant delays with different packet sizes and approaches G-McMAC when we get closer to the stability point of G-McMAC. However, with small packets the difference is remarkable and SYN-MAC introduces over twice as large delays as G-McMAC. Furthermore, performance of MMAC is significantly worse already with small packet sizes and access delay increases linearly when the packet

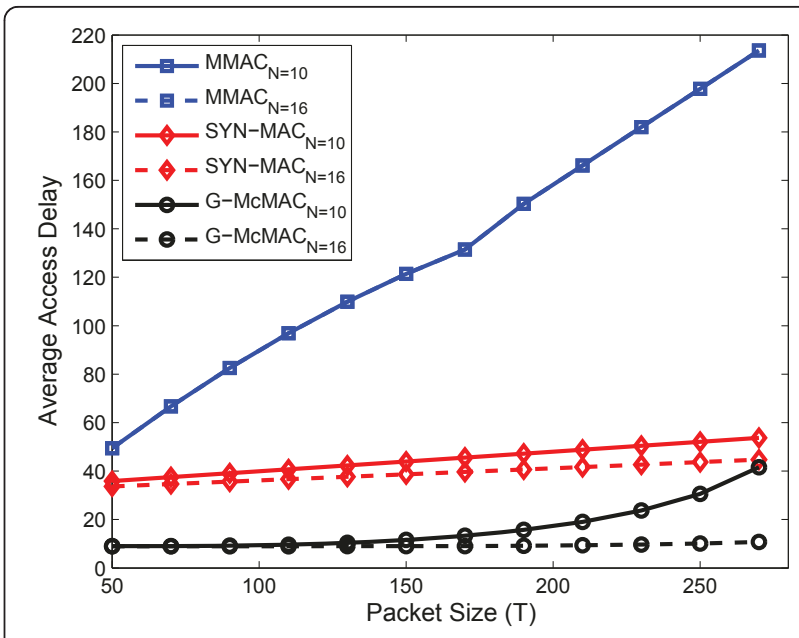

Figure 8 Average access delays as a function of packet size $(g$ $=0.04$ )

size grows. As the packet size is increased, delay of MMAC grows constantly and the difference compared with other protocols enhances. In this case, the number of channels does not have any impact on the delay of MMAC since $T_{\text {atim }} \leq 2 \omega$. As the results imply, delay of MMAC is heavily affected by the chosen packet size whereas G-McMAC and SYN-MAC offer relatively constant delays with different packet sizes. To summarize, G-McMAC achieves the best performance in general while SYN-MAC performs better with large packet sizes since it does not suffer from stability problems as quickly.

Different multi-channel approaches are not equally affected by the arrival rate as can be seen in Figure 9, where expected delays are depicted as a function of arrival rate. G-McMAC performs remarkably well while

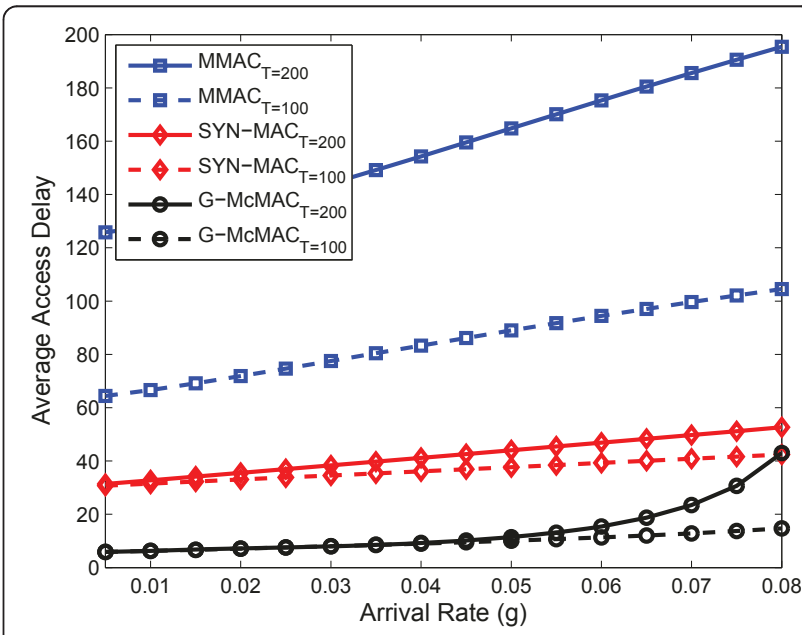

Figure 9 Average access delays as a function of arrival rate $(N$ = 16). 
arrival rates are small. However, as the arrival rate is increased, the difference in delay between G-McMAC and SYN-MAC diminishes when approaching to the stability point of G-McMAC. Nevertheless, G-McMAC achieves lower access delays regardless of the arrival rate given that a finite delay can be found for GMcMAC. However, the performance of MMAC is significantly worse already with low arrival rates since only a small number of successful negotiations can be carried out during the very short contention period and consequently, MMAC can not fully utilize the capacity of the multi-channel system. G-McMAC outperforms other protocols again while SYN-MAC achieves lower average access delays than MMAC.

As stated previously, the third critical parameter is the amount of available channels. According to our results shown in Figure 10, the performance of MMAC seems to be constant regardless of the number of channels when the packet size is small. This is because of the fact that when packet size is 100 the length of the contention period is 25 and hence, only a small amount of successful negotiations can be performed and MMAC does not exploit all the available channels. In other words, the performance is bounded by the length of ATIM windows rather than the number of channels. Moreover, even though the performance of SYN-MAC depends on the number of channels, the delay becomes close to constant quickly as the number of channels is increased. Nevertheless, SYN-MAC outperforms MMAC always. Finally, GMcMAC gives the lowest delays regardless of the number of channels and the performance saturates quickly with these parameters. However, it should be noted that GMcMAC does not achieve finite average access delays if $g$ $=0.04$ and $N \leq 6$ while $T=100$ or $N \leq 8$ while $T=200$.

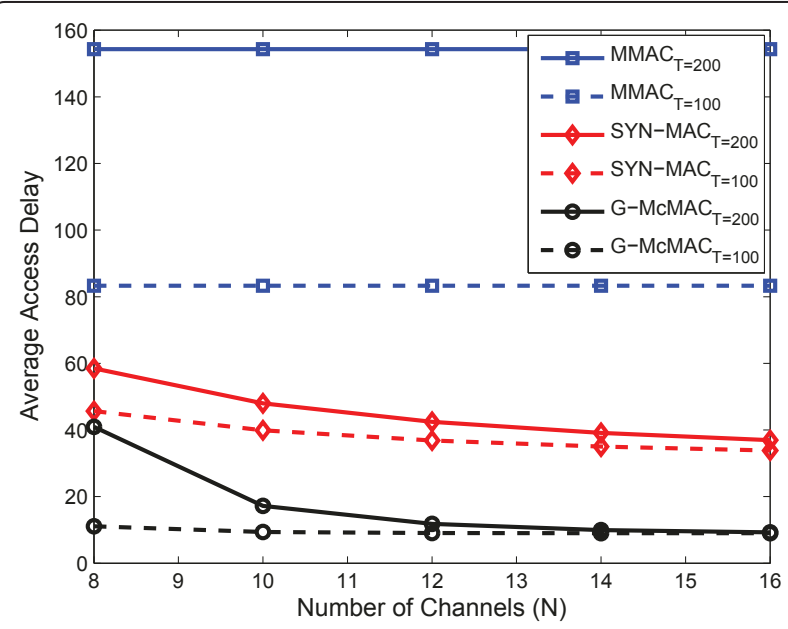

Figure 10 Average access delays as a function of number of channels $(g=0.04)$
The results infer that G-McMAC outperforms other protocols in terms of delay in all of the cases while it is stable.

\section{B. Throughput analysis}

Next, we study the impact of critical parameters on the throughput of different multi-channel MAC approaches. We begin with the effect of arrival rate and Figure 11 shows the protocols' throughputs as a function of packet arrival rate for two different packet sizes. In the case of small arrival rates G-McMAC outperforms other protocols clearly. However, the achieved gain depends on the chosen packet size and arrival rate. With these parameters, MMAC provides the smallest throughputs regardless of the arrival rate. On the other hand, SYNMAC will surpass G-McMAC in terms of throughput in all of the cases eventually when approaching the stability point of G-McMAC. For example, SYN-MAC will give better throughput than G-McMAC if $T=200, N=16$ and $g \geq 0.13$. As a conclusion, G-McMAC achieves better throughput than SYN-MAC especially when we have small or moderate arrival rates. The main reasons for this are that G-McMAC neither utilizes fixed contention periods such as SYN-MAC nor exploits periodic hopping patterns. Nevertheless, SYN-MAC will offer the highest throughputs in case of high arrival rates and small packets.

Naturally, throughput of multi-channel systems is dependent upon the number of available channels. Figure 12 demonstrates how the number of channels affects different multi-channel MAC approaches with a low packet arrival rate $g=0.04$. With these parameters, GMcMAC offers the highest throughput regardless of the amount of channels and once again, MMAC gives constant throughput due to the short ATIM window. However, now MMAC can offer higher throughputs than

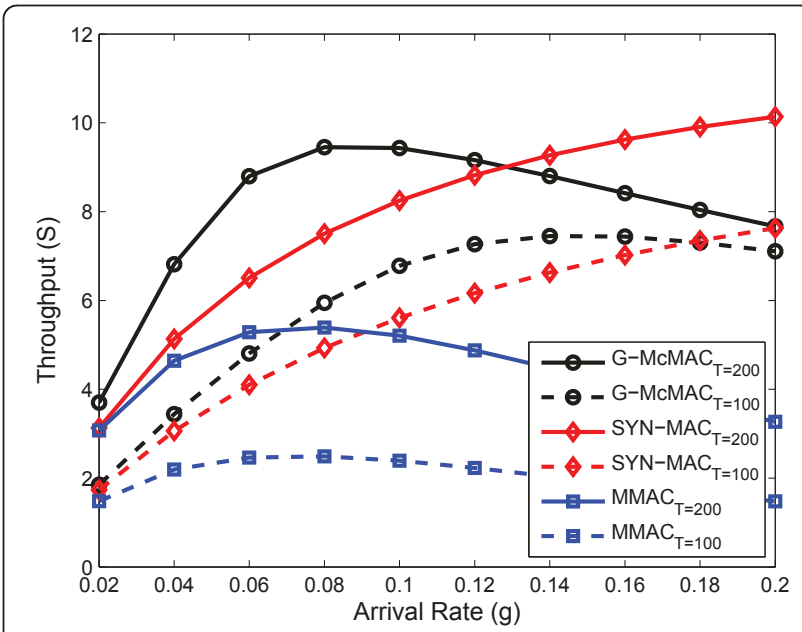

Figure 11 Throughputs as a function of arrival rate $(N=16)$ 


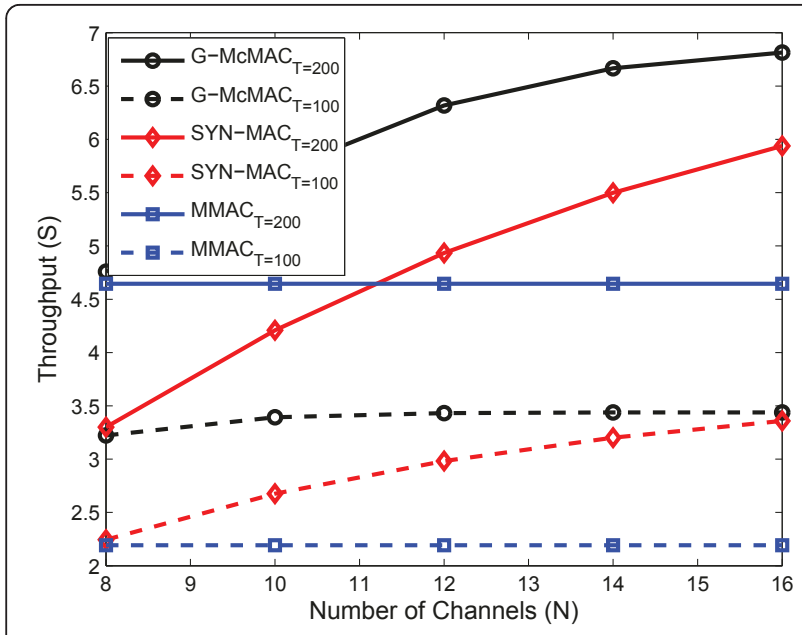

Figure 12 Throughputs as a function of channels $(g=0.04)$

SYN-MAC if the amount of available channels is small and $T=200$. The reason behind this is the cyclic hopping pattern of SYN-MAC which may cause silent periods during the operation. Nevertheless, SYN-MAC will achieve higher throughput than MMAC if the number of available channels is large. In case of small arrival rates SYN-MAC will also achieve as good performance as G-McMAC in terms of throughput if the number of channels is increased enough, even though average access delays of SYN-MAC are significantly higher as discussed previously.

On the other hand, with high arrival rates the situation is different as shown in Figure 13. In this case, the throughputs of SYN-MAC and G-McMAC grow linearly as the number of available channels is increased while MMAC gives constant throughput regardless of the amount of channels. However, now SYN-MAC

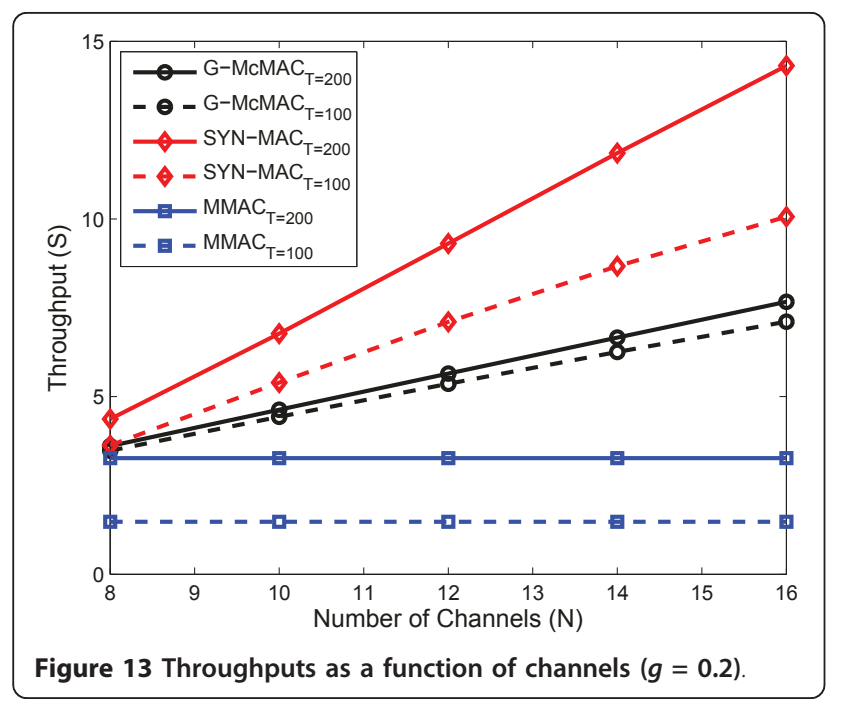

outperforms G-McMAC especially when the number of channels is large. The results imply that with these parameters the negotiation process of G-McMAC restricts the performance of the protocol since similar throughputs are achieved with different packet sizes. That is to say, with high packet arrival rates the common control channel will be occupied all the time which causes the performance to saturate. Since the negotiation process of SYN-MAC is shorter and carried out on each channel in turn, the performance continues to improve and the capacity of multi-channel systems can be fully exploited in case of high arrival rates and small packets.

We also studied the impact of different packet sizes on the throughputs and the results are presented in Figures 14 and 15 for $g=0.04$ and $g=0.2$, respectively. In general, the throughput of MMAC grows as a function of packet size due to the assumption of fixed and optimal packet sizes. Regardless of this fact SYN-MAC and G-McMAC outperform MMAC in case of small packets and small or moderate arrival rates. On the other hand, MMAC will eventually surpass other protocols since the performances of G-McMAC and SYN-MAC saturate at some point as the packet size is increased. Furthermore, G-McMAC gives better throughput than SYN-MAC with low packet arrival rates whereas SYN-MAC achieves similar performance with higher arrival rates. We can also see the impact of $P_{\text {block }}^{c}$ in Figure 15 since the performance of MMAC is constant when $N=10$ while it continues to improve when $N=16$. Nevertheless, it should be noted that in practice it may not be possible to predetermine optimal cycle structures for MMAC since packet sizes may be variable. This would naturally deteriorate the performance of MMAC. Moreover, average access delays of MMAC are many times worse than that of G-McMAC and the difference grows

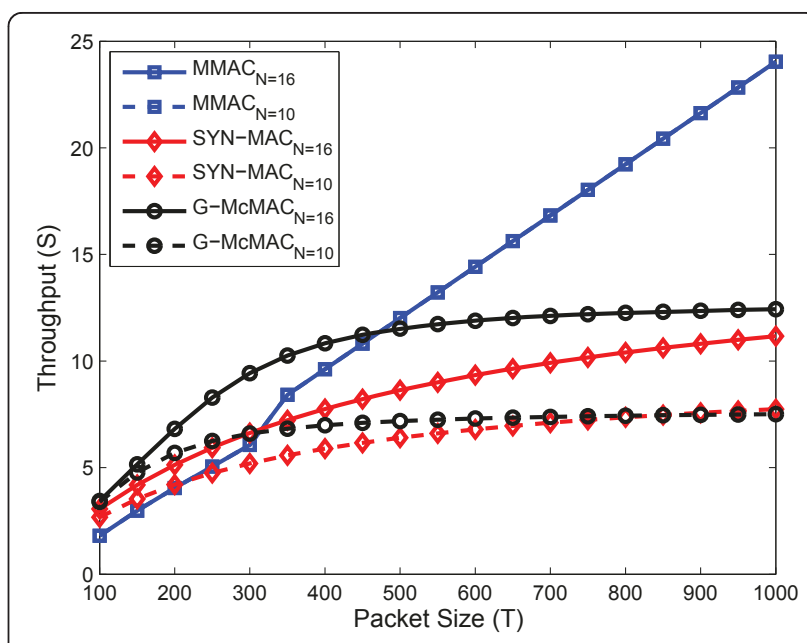

Figure 14 Throughputs as a function of packet size $(g=0.04)$ 


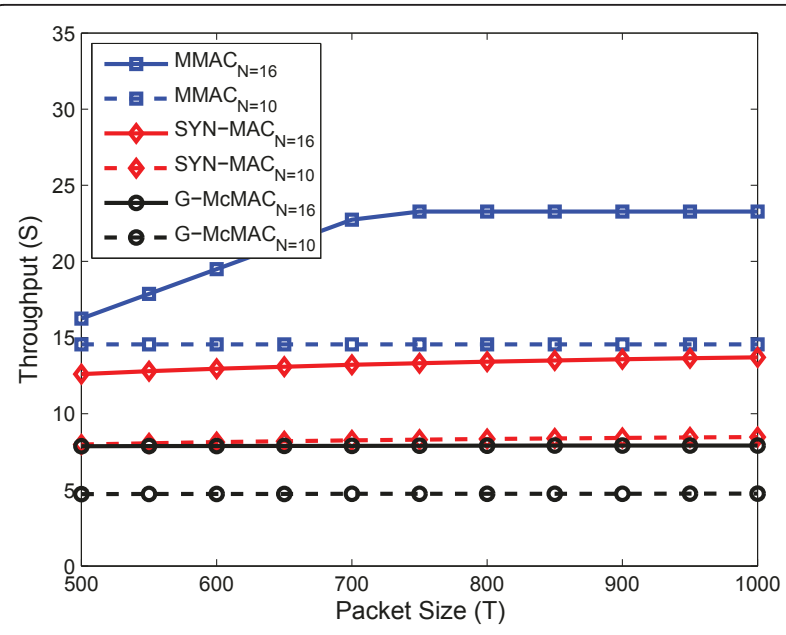

Figure 15 Throughputs as a function of packet size $(g=0.2)$.

as the packet size is increased, which makes MMAC unsuitable for delay-sensitive applications.

We conclude that G-McMAC achieves highest throughputs in case of small or moderate packet arrival rates while packets are small. On the other hand, SYNMAC outperforms other approaches in case of small packets and high packet arrival rates. Finally, MMAC provides the best performance with respect to throughput when the packets are large. However, MMAC causes very high latencies in general. Our delay analysis undoubtedly shows that G-McMAC outperforms other protocols clearly in terms of delay.

\section{System stability}

When embarking on any wireless communication design, it is essential to understand the operation region of the used MAC protocol to ensure system stability. Figure 16

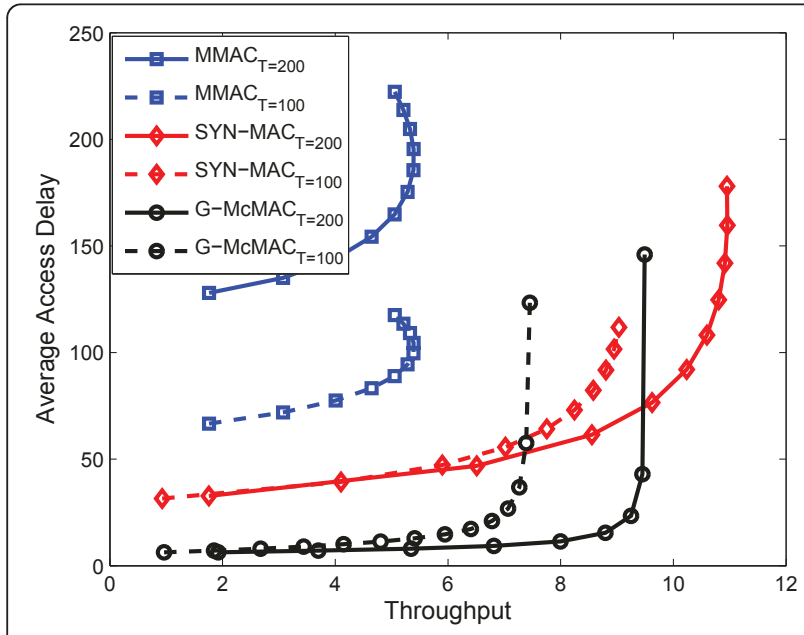

Figure 16 Delay as a function of throughput $(N=16)$. illustrates delay-throughput curves of MMAC, SYNMAC and G-McMAC. Evidently, MMAC performs the worst since it induces high latencies and becomes unstable when the throughput is low. After this point, the throughput of MMAC starts to decrease while delay continues to grow. On the other hand, we can see that G-McMAC clearly outperforms SYN-MAC by offering lower delays in general. However, G-McMAC becomes unstable before SYN-MAC and in fact after the stability point of G-McMAC the throughput of SYN-MAC still continues to improve. Based on this observation, we conclude that to minimize access delay, G-McMAC should be used. Whereas, if it is important to maximize throughput at the expense of access delays, SYN-MAC should be exploited.

\section{Conclusions}

In this paper, the performance of multi-channel MAC protocols in ad hoc networks was studied with respect to two important QoS parameters, delay and throughput. We deduced average access delays and throughputs for different multi-channel MAC approaches in closedform by considering Poisson arrivals. Theoretical results were verified by simulations for each of the considered protocols. Throughput and delay analyses were given in terms of critical system parameters such as number of available channels, arrival rate and packet sizes. We conclude that Generic Multi-channel MAC (G-McMAC) consistently outperforms other protocols with respect to delay. G-McMAC also achieves higher throughputs in some cases compared with other approaches, whereas, in some cases other approaches will achieve better throughput. Moreover, the low stability point of GMcMAC may be a problem for some applications and in those cases other approaches should be used. Presented results can be exploited to study the performance and suitability of different multi-channel MAC approaches for different wireless applications and to guide system design.

\section{APPENDIX A: Probabilities for G-MCMAC}

Performance evaluation of random access schemes has been traditionally carried out by exploiting busy period analysis in which the average busy time $\bar{B}$ and average idle time $\bar{I}$ are used for determining the characteristics of various schemes. In this appendix we consider GMcMAC and derive the following probabilities using the busy period analysis [19]: $P_{\mathrm{s}}$ is the probability of successful transmission, $P_{\mathrm{c}}$ is the probability of collision and $P_{\mathrm{b}}$ is the probability that the control channel is sensed busy. We model multi-channel communications with a Markov chain. States represent the number of occupied data channels such that we have $N-1$ data 
channels in total. Hence, the probability that all the channels are occupied can be found using the Erlang B formula [21] and is given by

$$
P_{\mathrm{ocC}}=\frac{\frac{G^{N-1}}{(N-1) !}}{\sum_{i=0}^{N-1} \frac{G^{i}}{i !}},
$$

where $G=g T$. To find out the probabilities, we need to derive the average idle and busy periods. For a start, the average idle period consists of $k-1$ times no arrivals and at least one arrival in the last slot. Hence, the average idle time $\bar{I}$ is

$$
\bar{I}=\frac{\tau}{1-e^{-g \tau}} .
$$

Next, we derive the average busy period which is defined as follows. The length of the busy period consists of $k$ transmission periods if there is at least one arrival in the last $k-1$ slots and no arrival in the last slot. Moreover, in the case of G-McMAC each busy period lasts $3 \tau+\tau$. Consequently, we find out the average busy period of G-McMAC as follows

$$
\bar{B}=\frac{4 \tau}{\mathrm{e}^{-g \tau}} .
$$

In one cycle, the number of possible time slots for successful transmission is $\bar{I} / \tau$ given that there is a packet arrival. Consequently, by taking into account the probability that all the channels are occupied we have

$$
\begin{aligned}
P_{\text {s }} & =P\{\text { success }\} \\
& =\frac{\bar{I} / \tau}{(\bar{I}+\bar{B}) / \tau} \cdot\left(1-P_{\text {occ }}\right) \\
& =\frac{e^{-g \tau}}{4-3 e^{-g \tau}} \cdot\left(1-P_{\text {occ }}\right) .
\end{aligned}
$$

Similarly, the amount of busy slots, i.e. channel is sensed busy and packet transmission delayed, is $\bar{B} \cdot 2 /(3 \tau)$. Now, we can derive the probability that the channel is sensed busy as follows

$$
\begin{aligned}
P_{\mathrm{b}} & =P\{\text { busy }\} \\
& =\frac{\bar{B} \cdot 2 /(3 \tau)}{(\bar{I}+\bar{B}) / \tau}+P_{\mathrm{s}} \cdot P_{\text {occ }} \\
& =\frac{3\left(1-\mathrm{e}^{-g \tau}\right)}{4-3 \mathrm{e}^{-g \tau}}+P_{\mathrm{s}} \cdot P_{\mathrm{occ}} .
\end{aligned}
$$

In the case of G-McMAC, the amount of collided packets is $\bar{B} / 4 \tau$. Hence, we can formulate the probability for packet collisions as

$$
\begin{aligned}
P_{\mathrm{C}} & =P\{\text { collision }\} \\
& =\frac{\bar{B} / 3 \tau}{(\bar{I}+\bar{B}) / \tau} \\
& =\frac{1-\mathrm{e}^{-g \tau}}{4-3 \mathrm{e}^{-g \tau}} .
\end{aligned}
$$

And clearly,

$$
\begin{aligned}
P_{\mathrm{s}}+P_{\mathrm{b}}+P_{\mathrm{c}} & =\frac{\mathrm{e}^{-g \tau}}{4-3 e^{-g \tau}}-P_{\mathrm{s}} \cdot P_{\mathrm{occ}} \\
& +\frac{3\left(1-\mathrm{e}^{-g \tau}\right)}{4-3 e^{-g \tau}}+P_{\mathrm{s}} \cdot P_{\mathrm{occ}} \\
& +\frac{1-\mathrm{e}^{-g \tau}}{4-3 e^{-g \tau}} \\
& =1,
\end{aligned}
$$

as expected. We verified theoretical derivations of $P_{\text {occ }}$ by simulations and the results are presented in Figure 17. As we can see, theoretical results correspond to simulation results well.

\section{APPENDIX B: Probabilities for MMAC}

In this appendix, the probabilities for MMAC during infinite ATIM windows will be deduced. Naturally, the average idle time of MMAC is equal to the average time of G-McMAC given in Equation (A-2). In the case of ATIM windows, the negotiation process consists of one sensing and RTS/CTS message exchange with our notation. Therefore, the length of the busy period is $2 \tau+\tau$, and the average busy period is

$$
\bar{B}=\frac{3 \tau}{e^{-g \tau}} .
$$

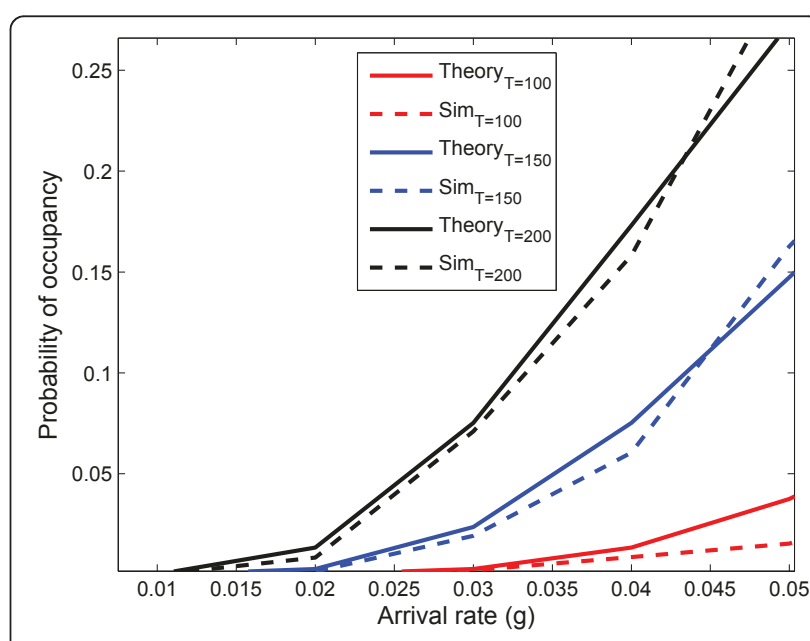

Figure 17 Theoretical and simulated results for $P_{\text {occ }}(N=10, \omega$ = 32) 


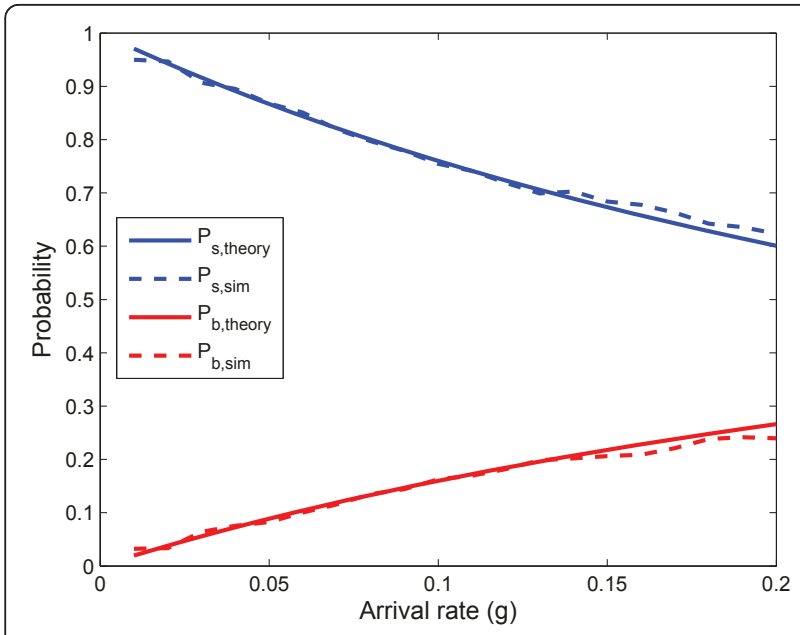

Figure 18 Theoretical and simulated results for $P_{\mathrm{s}}$ and $P_{\mathrm{b}}$.

Furthermore, the probability of successful transmission can now be derived as follows

$$
P_{\mathrm{s}}=\frac{\mathrm{e}^{-g \tau}}{\left(3-2 \mathrm{e}^{-g \tau}\right)} .
$$

In this case the amount of busy slots is $\bar{B} \cdot 2 /(3 \tau)$ and the probability that the channel is sensed busy is given by

$$
P_{\mathrm{b}}=\frac{2\left(1-\mathrm{e}^{-g \tau}\right)}{\left(3-2 \mathrm{e}^{-g \tau}\right)}
$$

Finally, collisions occur if the channel is sensed idle but more than one packet arrived during the last time slot. Now the amount of collided packets is $\bar{B} / 3 \tau$ and thus, we can formulate the following probability for packet collisions

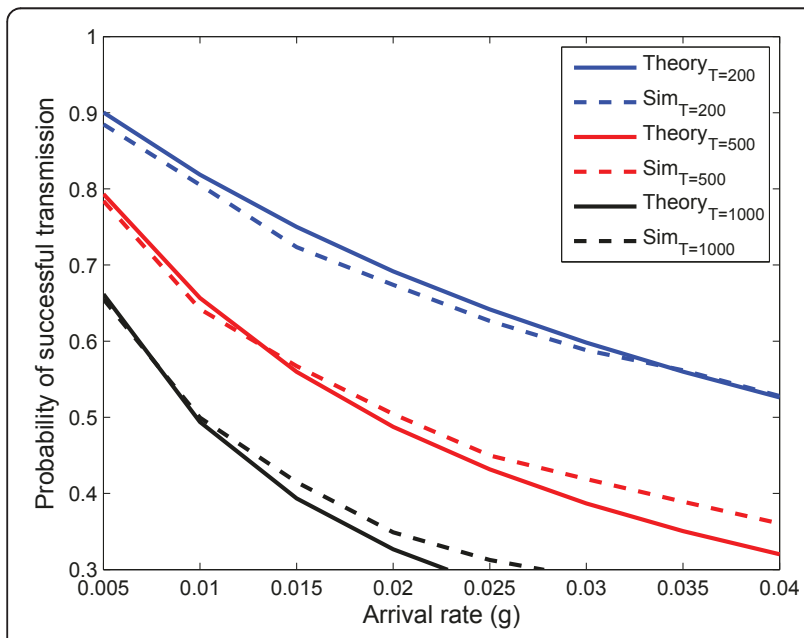

Figure 19 Theoretical and simulated results for $P_{\mathrm{s}}(N=10, \omega=$ $10)$.

$$
P_{\mathrm{C}}=\frac{1-\mathrm{e}^{-g \tau}}{\left(3-2 \mathrm{e}^{-g \tau}\right)} .
$$

Simulation results shown in Figure 18 attest the correctness of the theoretical results.

\section{APPENDIX C: Probabilities for SYN-MAC}

Finally, in this appendix we give the corresponding probabilities for SYN-MAC. Since SYN-MAC is modeled as a single channel system with reduced arrival rate the average idle time of SYN-MAC is equal to the average time of G-McMAC given in Equation (A-2) by substituting $\tau$ with $T_{\mathrm{s}}$. Moreover, in this case the length of a busy period is $T+T_{\mathrm{s}}$, where $T_{\mathrm{s}}=\omega \tau$, and thus, the average busy period of SYN-MAC is given by

$$
\bar{B}=\frac{T+T_{\mathrm{s}}}{\mathrm{e}^{-g_{\text {syn-mac }}}} .
$$

Furthermore, in one cycle the number of possible time slots for successful transmission is $\bar{I} / T_{\mathrm{s}}$ given that there is a packet arrival. Consequently, we have

$$
P_{\mathrm{s}}=\frac{\left(T_{\mathrm{s}} / T\right) \mathrm{e}^{-g \tau}}{1+\left(T_{\mathrm{s}} / T\right)-\mathrm{e}^{-g \tau}} .
$$

Similarly, the amount of busy slots is $\bar{B} \cdot\left(\left(T+T_{\mathrm{s}}\right) / T_{\mathrm{s}}-1\right) /\left(T+T_{\mathrm{s}}\right)$. As previously, we can now derive the probability that the channel is sensed busy as follows

$$
P_{\mathrm{b}}=\frac{1-\mathrm{e}^{-g \tau}}{1+\left(T_{\mathrm{s}} / T\right)-\mathrm{e}^{-g \tau}} .
$$

In the case of SYN-MAC, the amount of collided packets is $\bar{B} /\left(T+T_{\mathrm{s}}\right)$. Hence, we can formulate the probability for packet collisions as

$$
P_{\mathrm{C}}=\frac{\left(T_{\mathrm{s}} / T\right)\left(1-\mathrm{e}^{-g \tau}\right)}{1+\left(T_{\mathrm{s}} / T\right)-\mathrm{e}^{-g \tau}} .
$$

Once again, we verified theoretical derivations by simulations and the results are presented in Figure 19.

\section{Acknowledgements}

This research work is supported by TEKES (Finnish Funding Agency for Technology and Innovation) as part of the Wireless Sensor and Actuator Networks for Measurement and Control (WiSA-II) program.

\section{Competing interests}

The authors declare that they have no competing interests.

Received: 28 January 2011 Accepted: 22 September 2011 Published: 22 September 2011

\section{References}

1. A Goldsmith, S Wicker, Design Challenges for Energy-Constrained Ad Hoc Wireless Networks. IEEE Wireless Communications. 9(4), 8-27 (2002). doi:10.1109/MWC.2002.1028874 
2. T Bheemarjuna Reddy, I Karthigeyan, BS Manoj, C Siva Ram Murthy, Quality of Service Provisioning in Ad hoc Wireless Networks: A Survey of Issues and Solutions. Ad Hoc Networks. 4, 83-124 (2006)

3. L Kleinrock, F Tobagi, Packet Switching in Radio Channels: Part I-Carrier Sense Multiple-Access Modes and Their Throughput-Delay Characteristics. IEEE Transactions on Communications. 23(12), 1400-1416 (1975). doi:10.1109/TCOM.1975.1092768

4. Y Yang, T-S Yum, Delay Distributions of Slotted ALOHA and CSMA. IEEE Transactions on Communications. 51(11), 1846-1857 (2003). doi:10.1109/ TCOMM.2003.819201

5. G Bianchi, Performance Analysis of the IEEE 802.11 Distributed Coordination Function. IEEE Journal on Selected Areas in Communications. 18(3), 535-547 (2000). doi:10.1109/49.840210

6. J Mo, H-S So, J Walrand, Comparison of Multichannel MAC Protocols. IEEE Transactions on Mobile Computing. 7(1), 50-65 (2008)

7. S-L Wu, C-Y Lin, Y-C Tseng, J-L Sheu, A New Multi-channel MAC Protocol with On-demand Channel Assignment for Multi-hop Mobile Ad Hoc Networks. International Symposium on Parallel Architectures, Algorithms and Networks, I-SPAN 2000 232-237 (2000)

8. A Bachir, M Dohler, T Watteyne, K Leung, MAC Essentials for Wireless Sensor Networks. IEEE Communications Surveys Tutorials. 12(2), 222-248 (2010)

9. J So, NH Vaidya, Multi-channel Mac for Ad Hoc Networks: Handling Multichannel Hidden Terminals Using a Single Transceiver, in 5th ACM International Symposium on Mobile Ad Hoc Networking and Computing, MobiHoc '04, 222-233 (2004)

10. C Cordeiro, K Challapali, C-MAC: A Cognitive MAC Protocol for MultiChannel Wireless Networks, in 2nd IEEE International Symposium on New Frontiers in Dynamic Spectrum Access Networks, DySPAN '07, 147-157 (2007)

11. A Tzamaloukas, J Garcia-Luna-Aceves, Channel-Hopping Multiple Access, in IEEE International Conference on Communications, ICC 2000. 1, 415-419 (2000)

12. Y Kondareddy, P Agrawal, Synchronized MAC Protocol For Multi-Hop Cognitive Radio Networks, in IEEE International Conference on Communications, ICC 2008, 3198-3202 (2008)

13. Y Kim, H Shin, H Cha, Y-MAC: An Energy-Efficient Multi-channel MAC Protocol for Dense Wireless Sensor Networks, in International Conference on Information Processing in Sensor Networks, IPSN '08, 53-63 (2008)

14. HW So, J Walrand, J Mo, McMAC: A Parallel Rendezvous Multi-Channel MAC Protocol. IEEE Wireless Communications and Networking Conference, WCNC '07 334-339 (2007)

15. P Bahl, R Chandra, SSCH: Slotted Seeded Channel Hopping for Capacity Improvement in IEEE 802.11 Ad-Hoc Wireless Networks, in 10th ACM International Conference on Mobile Computing and Networking, MobiCom '04, 216-230 (2004)

16. J Li, Z Haas, M Sheng, Y Chen, Performance Evaluation of Modified IEEE 802.11 MAC for Multi-Channel Multi-Hop Ad Hoc Network, in 17th International Conference on Advanced Information Networking and Applications, AINA '03, 312-317 (2003)

17. T Luo, M Motani, V Srinivasan, CAM-MAC: A Cooperative Asynchronous Multi-Channel MAC Protocol for Ad Hoc Networks, in 3rd International Conference on Broadband Communications, Networks and Systems, BROADNETS '06, 1-10 (2006)

18. S Nethi, J Nieminen, R Jäntti, Exploitation of Multi-Channel Communications in Industrial Wireless Sensor Applications: Avoiding Interference and Enabling Coexistence, in Wireless Communications and Networking Conference, WCNC'11, 345-350 (2011)

19. R Rom, M Sidi, Multiple Access Protocols: Performance and Analysis, (SpringerVerlag, 1990)

20. L Hanzo-II, R Tafazolli, A Survey of QoS Routing Solutions for Mobile Ad Hoc Networks. IEEE Communications Surveys \& Tutorials 9(2), 50-70 (2007)

21. AK Erlang, Solutions of some Problems in the Theory of Probabilities of Significance in Automatic Telephone Exchanges. Elektroteknikeren. 13, 138-155 (1917)

doi:10.1186/1687-1499-2011-108

Cite this article as: Nieminen and Jäntti: Delay-throughput analysis of multi-channel MAC protocols in ad hoc networks. EURASIP Journal on Wireless Communications and Networking 2011 2011:108.

\section{Submit your manuscript to a SpringerOpen ${ }^{\mathcal{O}}$ journal and benefit from:}

- Convenient online submission

- Rigorous peer review

- Immediate publication on acceptance

- Open access: articles freely available online

- High visibility within the field

- Retaining the copyright to your article

Submit your next manuscript at $\gg$ springeropen.com 